\title{
Genetic strain variations in the metaphase-II phenotype of mouse oocytes matured in vivo or in vitro
}

\author{
Elena Ibáñez ${ }^{1}$, Alexandra Sanfins ${ }^{2,3}$, Catherine M H Combelles $^{2}$, Eric W Overström ${ }^{1,2}$ \\ and David F Albertini ${ }^{2}$ \\ ${ }^{1}$ Department of Biomedical Sciences, Tufts University School of Veterinary Medicine, North Grafton, \\ Massachusetts 01536, USA, ${ }^{2}$ Department of Anatomy and Cellular Biology, Tufts University School of Medicine, \\ Boston, Massachusetts 02111, USA and ${ }^{3}$ Instituto de Medicina Molecular, Unidade de Biologia da Reprodução, \\ Faculdade de Medicina de Lisboa, Lisbon, Portugal
}

Correspondence should be addressed to D F Albertini, Department of Molecular and Integrative Physiology, The University of Kansas Medical Center, 3901 Rainbow Boulevard, Kansas City, Kansas 66160-7401, USA; Email: dalbertini@kumc.edu

E Ibàñez is now at Departament de Biologia Cellular, Fisiologia i Immunologia, Facultat de Ciències, Universitat Autònoma de Barcelona, 08193 Bellaterra, Spain

C M H Combelles is now at Biology Department, McCardell Bicentennial Hall 350, Middlebury College, Middlebury, Vermont 05753, USA

E W Overström is now at Department of Biology and Biotechnology, Worcester Polytechnic Institute, 100 Institute Road, Worcester, Massachusetts 01609-2280, USA

\begin{abstract}
The interplay between genetic and epigenetic factors plays a central role in mammalian embryo production strategies that superimpose ex vivo or in vivo manipulations upon strain background characteristics. In this study, we examined the relationship between genetic background and the phenotypic properties of mouse metaphase-II (M-II) oocytes that were matured under in vivo (IVO) or in vitro conditions, either in a basal (IVM) or a supplemented (IVM + ) medium. Differences existed amongst inbred (C57BL/6), outbred (CF-1, Black Swiss, NU/NU) and hybrid lines (B6D2F1) induced to superovulate with regard to cytoplasmic microtubule organizing center (MTOC) number but not spindle size or shape, except for larger and asymmetrical spindles in Black Swiss oocytes. When oocytes were matured in culture, meiotic spindle and cytoplasmic phenotypic properties of M-II oocytes were affected relative to in vivo conditions and between strains. Specifically, measures of meiotic spindle size, shape, polar pericentrin distribution and cytoplasmic MTOC number all revealed characteristic variations. Interestingly, the overall reduction in cytoplasmic MTOC number noted upon IVM was concomitant with an overall increase in spindle and polar body size. Maturation under IVM + conditions resulted in a further decrease in cytoplasmic MTOC number, but spindle and polar body characteristics were intermediate between IVO and IVM. How these oocyte phenotypic properties of maternal origin may be linked to predictive assessments of fecundity remains to be established. Reproduction (2005) 130 845-855
\end{abstract}

\section{Introduction}

Despite the widespread usage of mice in the development of assisted reproductive technologies, and the availability of various genetic strains in which to assess fecundity determinants, surprisingly little is known about the genetic basis of oocyte quality. Genetic strain variations in mice with regard to in vitro fertilization (Kaleta 1977), cleavage rates (McLaren \& Bowman 1973, Luckett \& Mukherkee 1986), pH regulation (Steeves et al. 2001), parthenogenetic activation (Gao et al. 2004, Ibáñez et al. 2005) and preimplantation development (Suzuki et al. 1996) buttress the well-known relationship between fecundity and gen- etic background amongst hybrid F1, inbred and outbred lines (Silver 1995). Additionally, variations in hormone sensitivity influence ovulation rate amongst different mouse strains (Spearow 1988, Spearow et al. 1999) and the rate of meiotic maturation in vivo or in vitro can vary depending on genetic background (Polanski 1986, 1997). Thus, it seems likely that some of the genetic factors that specify embryo quality find their roots in maternal preconceptional processes associated with oogenesis and ovulation. Of note in this regard is the potential interplay of genetic and environmental influences during oocyte maturation. 
It is well established that the environment to which the oocyte is exposed during maturation influences the developmental potential of the resulting embryos and, in many mammalian species, in vitro matured oocytes exhibit lower rates of embryonic development than in vivo matured oocytes after fertilization (Eppig \& O'Brien 1998, Niemann \& Wrenzycki 2000, Moor \& Dai 2001, Trounson et al. 2001). Additional properties distinguish in vitro from in vivo matured oocytes, such as mitochondria and ATP content (Combelles \& Albertini 2003, Nishi et al. 2003), protein synthesis (Schultz et al. 1978), translation and transcription patterns (Eichenlaub-Ritter \& Peschke 2002, Rodriguez \& Farin 2004), glutathione content (de Matos et al. 2003) and imprinting/methylation patterns in embryos derived from in vivo and in vitro oocytes (Lucifero et al. 2002). Whether such potential determinants of oocyte quality result from genetic predisposition or environmental sensitivities remains unknown.

Recently we have shown that the temporal progression of cytoplasmic and nuclear remodeling events were distinctly different in in vivo matured oocytes compared with oocytes matured under basal in vitro conditions in the outbred CF-1 mouse strain (Sanfins et al. 2003, 2004), further supporting the notion that in vivo produced ova are generally of superior quality with respect to production of healthy embryos and offspring. It is well established that during in vitro maturation, although nuclear maturation takes place, complete cytoplasmic maturation is not assured (Eppig et al. 1996). Therefore, important factors must be added to the culture medium in an attempt to recapitulate the follicular environment and improve cytoplasmic maturation. With this in mind, we began to design a supplemented maturation medium $($ IVM + ), based on several reports addressing the positive effects of certain components on improving cytoplasmic maturation and, consequently, oocyte maturation efficiency. Specifically, addition of D-glucosamine as a substrate for hyaluronic acid (HA) synthesis improves cumulus expansion (Chen et al. 1990, Dell'Aquila et al. 2004). Cysteine is a critical factor for the synthesis of glutathione (de Matos et al. 1996), which has been shown to be important in protecting the oocyte from oxidative damage and essential, after fertilization, for sperm chromatin descondensation, male pronuclear formation and subsequent development to the blastocyst stage (Perreault et al. 1988, de Matos et al. 1995). Ascorbic acid, another anti-oxidant, prevents follicular apoptosis in mouse follicles and increases developmental competence of in vitro matured oocytes (Eppig et al. 2000, Tatemoto et al. 2001). Finally, follicle-stimulating hormone $(\mathrm{FSH})$ has been shown to stimulate de novo $\mathrm{HA}$ synthesis and cumulus expansion (Eppig 1979, 1980) and is routinely added to culture media to improve maturation and oocyte developmental competence (Merriman et al. 1998, Eppig et al. 2000).

The aim of the present study was to analyze the potential effect of both the genetic background and the maturation conditions on the metaphase-II (M-II) phenotype of mouse oocytes. With this aim, M-II oocytes from five different strains of mice were obtained either after superovulation (IVO) or by culture in a basal (IVM) or in a supplemented (IVM +) maturation medium, and their phenotypic properties in terms of meiotic spindle size and organization, number of cytoplasmic microtubule organizing centers (MTOCs) and first polar body characteristics were compared. The results obtained reveal distinct phenotypic variations in the organization of the microtubule-centrosome complex based upon genetic background that are subjected to epigenetic changes during in vitro maturation.

\section{Materials and Methods \\ Collection, culture and fixation of mouse oocytes}

Mouse strains used as oocyte donors were B6D2F1 $(\mathrm{C} 57 \mathrm{BL} / 6 \times \mathrm{DBA} / 2), \mathrm{C} 57 \mathrm{BL} / 6$ and CF-1, as common representatives of hybrid, inbred and outbred strains respectively. In addition, oocytes collected from Black Swiss and from immunodeficient homozygous nude (NU/NU) females, two other outbred strains that were being used at the time for other work by our group, were also included in the study. B6D2F1, C57BL/6, CF-1 and NU/NU female mice were obtained from Charles River Laboratories (Wilmington, MA, USA) and Black Swiss mice were obtained from Taconic (Germantown, NY, USA). All animals were handled according to the Guide for Care and Use of Laboratory Animals (National Academy of Science 1996) and to protocols approved by the Tufts University Institutional Animal Care and Use Committee. Animals were maintained on a $14 \mathrm{~h}$ light: $10 \mathrm{~h}$ darkness photoperiod under constant temperature and relative humidity conditions, and food and water were provided ad libitum.

In vivo matured oocytes (IVO) were obtained from mice (8-12 weeks old) previously injected with 5 IU equine chorionic gonadotropin (eCG; Calbiochem, San Diego, CA, USA) to stimulate follicular development, followed $48 \mathrm{~h}$ later by $5 \mathrm{IU}$ human chorionic gonadotropin (hCG; Calbiochem) to induce ovulation. Oocytes were collected from oviducts $16 \mathrm{~h}$ after hCG injection in a collection medium consisting of Hepes-buffered Eagle's minimum essental medium (MEM) with Hanks' salts (Gibco-Invitrogen Life Technologies, Grand Island, NY, USA) supplemented with $100 \mathrm{IU}$ penicillin/ml, $100 \mu \mathrm{g}$ streptomycin $/ \mathrm{ml}$ and $0.3 \%(\mathrm{w} / \mathrm{v})$ bovine serum albumin (BSA; Sigma, St Louis, MO, USA). The mean number ( \pm S.E.M.) of ovulated oocytes recovered per female was as follows: $\quad \mathrm{B} 6 \mathrm{D} 2 \mathrm{~F} 1=20.3 \pm 0.3, \quad \mathrm{C} 57 \mathrm{BL} / 6=16.7 \pm 2.9$, Black Swiss $=13.9 \pm 1.1, \quad \mathrm{CF}-1=19.8 \pm 2.1 \quad$ and NU/NU $=27.9 \pm 1.3$ oocytes/female (15 females per strain). Following removal of cumulus cells by a 5 -min treatment with $150 \mathrm{U}$ bovine testicular hyaluronidase/ml (Sigma) in collection medium at room temperature, oocytes were immediately fixed and extracted for $30 \mathrm{~min}$ at $37^{\circ} \mathrm{C}$ in a microtubule stabilizing buffer $(0.1 \mathrm{~mol}$ 
PIPES/ml, pH 6.9, $5 \mathrm{mmol} \quad \mathrm{MgCl}_{2} \times 6 \mathrm{H}_{2} \mathrm{O} / \mathrm{ml}$ and $2.5 \mathrm{mmol} \mathrm{EGTA} / \mathrm{ml})$ containing $2 \%(\mathrm{v} / \mathrm{v})$ formaldehyde, $0.1 \%(\mathrm{v} / \mathrm{v})$ Triton X-100, $1 \mu \mathrm{mol}$ taxol$/ \mathrm{ml}, 10 \mathrm{U}$ aproti$\mathrm{nin} / \mathrm{ml}, 1 \mathrm{mmol}$ dithiothreitol $/ \mathrm{ml}$ and $50 \%$ (v/v) deuterium oxide (Messinger \& Albertini 1991). Fixed oocytes were stored at $4^{\circ} \mathrm{C}$ in a blocking solution of phosphate-buffered saline (PBS) containing 2\% (w/v) BSA, 2\% (w/v) powdered milk, 2\% (v/v) normal goat serum, $0.1 \mathrm{~mol}$ glycine $/ \mathrm{ml}$ and $0.01 \%(\mathrm{v} / \mathrm{v})$ Triton X-100 until further processing.

For in vitro maturation experiments, mice (8-12 weeks old) were injected $45-48 \mathrm{~h}$ earlier with $5 \mathrm{IU}$ eCG and cumulus-enclosed oocytes were collected by follicular puncture in collection medium. Immature oocytes surrounded by cumulus cells were selected and were cultured for $16 \mathrm{~h}$ in either a basal maturation medium (IVM) consisting of Eagle's MEM supplemented with Earle's salts (GibcoInvitrogen Life Technologies), $2 \mathrm{mmol}$ glutamine/ml, $0.23 \mathrm{mmol}$ pyruvate/ml, $100 \mathrm{IU}$ penicillin/ml, $100 \mu \mathrm{g}$ streptomycin $/ \mathrm{ml}$ and $0.3 \%(\mathrm{w} / \mathrm{v})$ BSA or in the same medium additionally supplemented with $0.5 \mathrm{mg}$ D-glucosamine $/ \mathrm{ml}$ (Sigma), $0.6 \mathrm{mmol}$ cysteine $/ \mathrm{ml}$ (Sigma), $0.02 \mu \mathrm{mol}$ ascorbic $\mathrm{acid} / \mathrm{ml}$ (Sigma) and $0.2 \mathrm{ng}$ ovine $\mathrm{FSH} / \mathrm{ml}$ (National Institute of Diabetes and Digestive and Kidney Diseases, Baltimore, MD, USA) (IVM +). All cultures were maintained in a humidified atmosphere of $5 \% \mathrm{CO}_{2}$ in air. After removing cumulus cells by gentle pipetting, oocytes were fixed and stored at $4{ }^{\circ} \mathrm{C}$ as described above until further processing.

\section{Processing of oocytes for fluorescence microscopy}

For each experiment, IVO, IVM and IVM + oocytes from the five strains of mice were processed in parallel for phenotypic evaluation by fluorescence microscopy. In this way, spindle size and morphology, chromatin and cytoplasmic MTOCs could be evaluated simultaneously by digital imaging in samples processed under identical conditions. Oocytes were incubated sequentially with primary and secondary antibodies for $1 \mathrm{~h}$ at $37^{\circ} \mathrm{C}$ with shaking, followed by three 15-min washes in blocking solution. First, MTOCs were labeled using a rabbit polyclonal anti-pericentrin antibody (Doxsey et al. 1994) followed by affinitypurified Texas Red-conjugated donkey anti-rabbit IgG (1:150; Jackson ImmunoResearch Laboratories Inc, West Grove, PA, USA). Secondly, microtubules were labeled by using a 1:1 mixture of mouse monoclonal anti- $\alpha$-tubulin and anti- $\beta$-tubulin antibodies (Sigma) at a 1:100 final dilution, followed by incubation with affinity-purified fluoresceinated donkey anti-mouse IgG (1:50, Jackson ImmunoResearch Laboratories, Inc.). Oocytes were then mounted in a $50 \%$ glycerol/PBS solution containing $0.2 \%$ (w/v) sodium azide (Sigma) and $1 \mu \mathrm{g}$ Hoechst 33258/ml (Polysciences Inc., Warrington, PA, USA) to label chromatin. Incubation of oocytes in secondary antibodies alone failed to yield detectable staining. Labeled oocytes were analyzed on a Zeiss IM-35 inverted microscope using $50 \mathrm{~W}$ mercury arc lamp and a $40 \times$ Neofluor objective. Digital images were collected with a Hamamatsu Orca ER digital camera (model C4742-95) interfaced with a MetaMorph Imaging System 5.0 (Universal Imaging Corp., Downington, PA, USA). A triple band pass dichroic and automated excitation filter wheel permitted collection of spatially registered images with minimal magnification distortion. Digital images of single oocytes within each experimented group were collected under identical exposure conditions for each of the fluors.

\section{Quantification of spindle size and cytoplasmic MTOC numbers}

Oocytes were first analyzed to determine meiotic status based upon previously reported criteria (Albertini 1992). Digital images were then obtained from M-II oocytes with integration times of 500-1000 ms for the fluorescein channel at subsaturation pixel intensities and thresholding, and spatial measurements were recorded with the MetaMorph Imaging System after calibration with a stage micrometer. Images with clear definition of spindle boundaries and of appropriate orientation were used to obtain measurements of spindle length (from pole to pole), spindle width at the equator and spindle pole width, and from these values the total area of the spindle was calculated for each oocyte. To account for half-spindle asymmetry, a formula was derived for determination of spindle area that would take into account differences between each half spindle (Sanfins et al. 2003).

For each group of oocytes, the organization of pericentrin material at the spindle poles was also analyzed, and characterized as solitary or multiple foci and c-shaped aggregates, as previously described (Carabatsos et al. 2000). Additionally, the number of cytoplasmic MTOCs was determined in each oocyte based on pericentrin-positive foci that were not spindle associated (Messinger \& Albertini 1991).

\section{Statistical analysis}

Experiments were performed in parallel for the five different mouse strains and the three maturation conditions and were repeated twice. The percentages of IVO, IVM and IVM + oocytes at $M$-II in the five strains analyzed were compared by Chi-square analysis. Two-way ANOVA by strain and maturation conditions (IVO, IVM, IVM + ) were used to compare spindle dimensions (length, width, pole width and area) and the number of cytoplasmic MTOCs. The Newman-Keul's multiple range test was used for post-hoc comparisons. $P<0.05$ was considered significant.

\section{Results}

\section{Meiotic competence differences between IVO, IVM and IVM + oocytes are strain specific}

The ability of oocytes to complete the first meiotic division and arrest at the M-II stage was analyzed in oocytes 


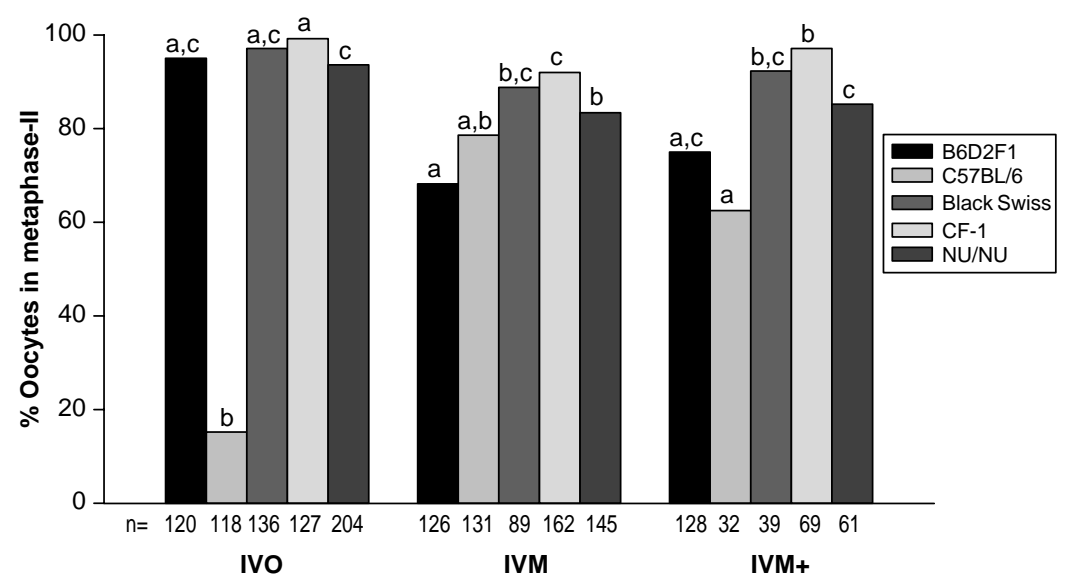

Figure 1 Maturation rates of oocytes from five strains of mice matured under in vivo (IVO) or in vitro (IVM and IVM + ) conditions. Data represent the combined results of two replicate experiments for each maturation treatment, and the total number of oocytes analyzed ( $n$ ) for each strain and treatment are indicated at the bottom of the columns. Different letters at the top of the columns represent significant differences $(P<0.05)$ across strains within each maturation treatment (Chisquare). matured under in vivo (IVO) or in vitro (IVM and IVM + ) conditions for each of the five strains of mice used in this study. As shown in Fig. 1, different extents of maturation were observed for each group. Under IVO conditions, all strains completed maturation except C57BL/6, which exhibited a significantly lower percentage of $\mathrm{M}$-II oocytes due to a delay in meiotic progression at the anaphase-telophase of meiosis I. Under IVM and IVM + conditions, lower maturation rates were observed for B6D2F1 and C57BL/6 oocytes respectively when compared with oocytes from the three outbred strains.

Overall, for all strains, the percentage of M-Il oocytes in IVM was significantly lower than in IVO $(P<0.05)$, except in the C57BL/6 group where a significantly higher

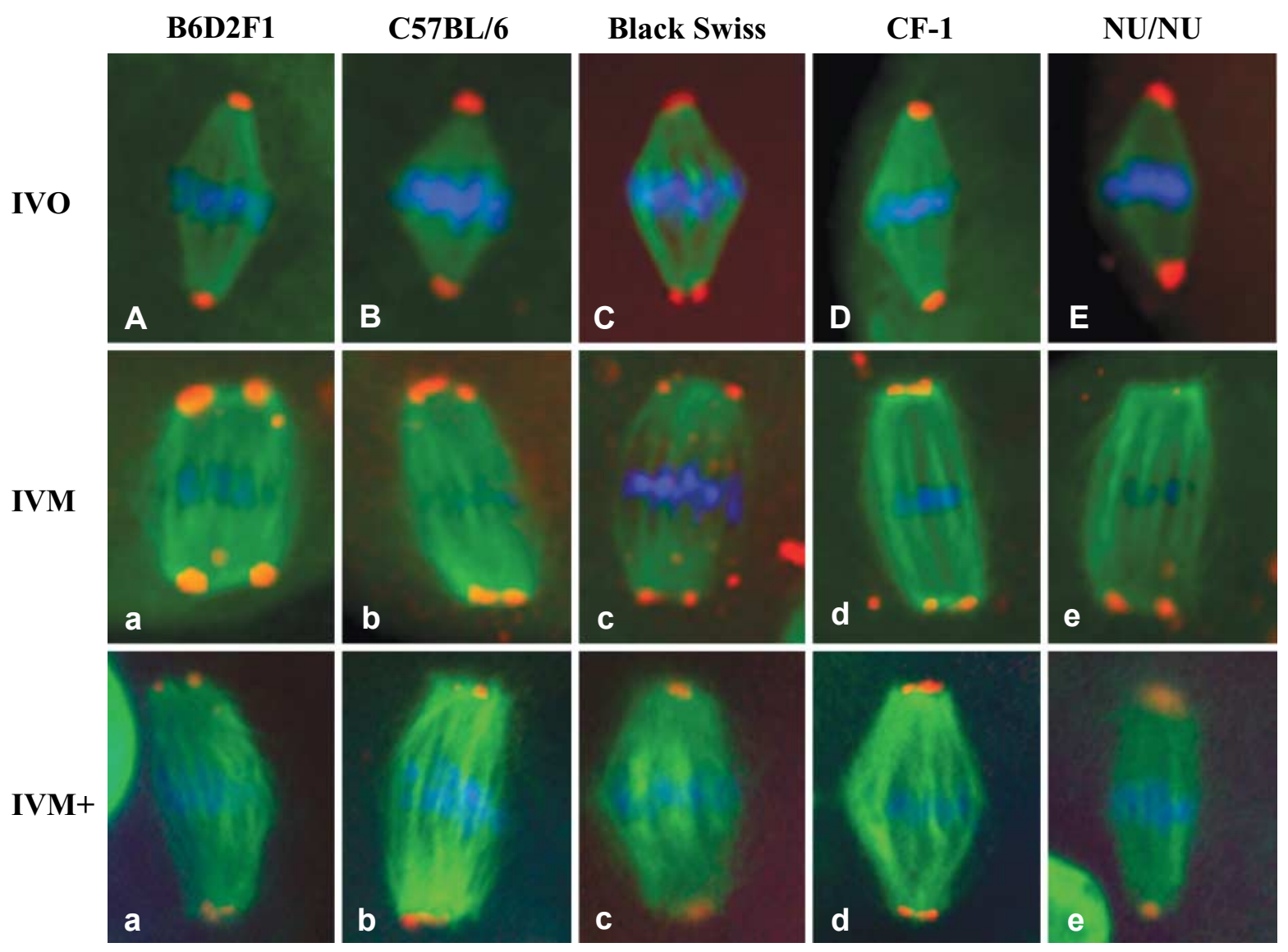

Figure $2 \mathrm{M}$-II spindles of (A-E) IVO, (a-e) IVM and $\left(\mathrm{a}^{\prime}-\mathrm{e}^{\prime}\right)$ IVM + oocytes in the five strains of mice studied. Tubulin (green), pericentrin (red) and chromosomes (blue) are depicted. Note the pointed/tapered-shaped spindles with solitary pericentrin foci at the poles of IVO oocytes (A-E), contrasting with the barrel-shaped spindle with fragmented pericentrin foci at the poles of IVM oocytes (a-e). IVM + oocytes exhibit big spindles but with focused pericentrin aggregates at the poles $\left(\mathrm{a}^{\prime}-\mathrm{e}^{\prime}\right)$. 
Table 1 Spindle dimensions in M-II oocytes from five different mouse strains collected after ovulation (IVO) or matured in vitro in a basal (IVM) or a supplemented (IVM + ) maturation medium. Values are means \pm S.E.M.

\begin{tabular}{|c|c|c|c|c|c|c|}
\hline \multirow[b]{2}{*}{ Maturation conditions } & \multirow[b]{2}{*}{ Spindle dimensions } & \multicolumn{5}{|c|}{ Strain of oocytes } \\
\hline & & B6D2F1 & $\mathrm{C} 57 \mathrm{BL} / 6$ & Black Swiss & CF-1 & $\mathrm{NU} / \mathrm{NU}$ \\
\hline \multirow[t]{5}{*}{ IVO } & Length $(\mu \mathrm{m})$ & $22.3 \pm 0.4$ & $23.1 \pm 0.5$ & $22.8 \pm 0.3$ & $22.4 \pm 0.5$ & $21.9 \pm 0.3$ \\
\hline & Width $(\mu \mathrm{m})$ & $11.5 \pm 0.2^{\mathrm{a}}$ & $11.9 \pm 0.5^{\mathrm{a}}$ & $14.2 \pm 0.4^{\mathrm{b}}$ & $11.8 \pm 0.3^{\mathrm{a}}$ & $11.7 \pm 0.3^{\mathrm{a}}$ \\
\hline & Pole width $(\mu \mathrm{m})$ & $3.6 \pm 0.1$ & $3.9 \pm 0.1$ & $4.3 \pm 0.2$ & $3.3 \pm 0.1$ & $3.7 \pm 0.1$ \\
\hline & Area $\left(\mu \mathrm{m}^{2}\right)$ & $168.6 \pm 3.9^{\mathrm{a}}$ & $182.8 \pm 6.8^{\mathrm{a}, \mathrm{b}}$ & $211.8 \pm 6.3^{\mathrm{b}}$ & $169.8 \pm 5.6^{\mathrm{a}}$ & $170.2 \pm 4.9^{\mathrm{a}}$ \\
\hline & $n$ & 29 & 21 & 30 & 33 & 31 \\
\hline \multirow[t]{5}{*}{ IVM } & Length $(\mu \mathrm{m})$ & $26.0 \pm 0.5^{a, b}$ & $25.3 \pm 0.6^{\mathrm{a}}$ & $27.6 \pm 0.7^{\mathrm{b}}$ & $24.6 \pm 0.4^{\mathrm{a}, \mathrm{c}}$ & $26.6 \pm 0.8^{a, b}$ \\
\hline & Width $(\mu \mathrm{m})$ & $13.5 \pm 0.6^{\mathrm{a}}$ & $13.1 \pm 0.4^{\mathrm{a}}$ & $16.9 \pm 0.5^{\mathrm{b}}$ & $12.8 \pm 0.4^{\mathrm{a}}$ & $12.8 \pm 0.5^{\mathrm{a}}$ \\
\hline & Pole width $(\mu \mathrm{m})$ & $9.3 \pm 0.4^{\mathrm{a}}$ & $6.6 \pm 0.3^{b}$ & $9.1 \pm 0.4^{\mathrm{a}, \mathrm{c}}$ & $6.7 \pm 0.3^{b}$ & $8.3 \pm 0.4^{\mathrm{c}}$ \\
\hline & Area $\left(\mu \mathrm{m}^{2}\right)$ & $300.2 \pm 14.2^{\mathrm{a}}$ & $249.7 \pm 9.8^{\mathrm{C}}$ & $360.3 . \pm 14.9^{b}$ & $241.3 \pm 8.5^{\mathrm{c}}$ & $287.4 \pm 18.8^{\mathrm{a}}$ \\
\hline & $n$ & 31 & 31 & 30 & 34 & 32 \\
\hline \multirow[t]{5}{*}{ IVM+ } & Length $(\mu \mathrm{m})$ & $24.6 \pm 0.8$ & $23.9 \pm 0.7$ & $26.2 \pm 0.9$ & $24.3 \pm 0.4$ & $24.8 \pm 0.5$ \\
\hline & Width $(\mu \mathrm{m})$ & $12.5 \pm 0.3^{\mathrm{a}}$ & $12.5 \pm 0.6^{\mathrm{a}, \mathrm{b}}$ & $12.6 \pm 0.4^{\mathrm{a}, \mathrm{b}}$ & $14.2 \pm 0.5^{\mathrm{b}}$ & $10.2 \pm 0.4^{c}$ \\
\hline & Pole width $(\mu \mathrm{m})$ & $6.9 \pm 0.4^{\mathrm{a}}$ & $5.3 \pm 0.5^{\mathrm{b}, \mathrm{c}}$ & $5.8 \pm 0.4^{\mathrm{b}}$ & $4.3 \pm 0.2^{\mathrm{C}}$ & $5.4 \pm 0.5^{\mathrm{b}, \mathrm{c}}$ \\
\hline & Area $\left(\mu \mathrm{m}^{2}\right)$ & $239.1 \pm 11.2$ & $214.9 \pm 14.0$ & $241.9 \pm 13.1$ & $225.9 \pm 8.1$ & $195.5 \pm 12.7$ \\
\hline & $n$ & 21 & 12 & 18 & 25 & 14 \\
\hline
\end{tabular}

Pole width indicates the mean width at the two spindle poles.

${ }^{\mathrm{a}-\mathrm{C}}$ Different superscripts denote significant differences $(P<0.05)$ across strains within the same maturation conditons for each parameter analyzed (ANOVA).

incidence of M-II oocytes was observed suggesting that the meiotic delay detected during IVO is overcome with IVM. There was no significant variation in the percentage of M-II oocytes between IVM + and IVM groups for any of the strains examined, and it is noteworthy that for all outbred lines (Black Swiss, CF-1 and NU/NU) the extent of maturation was equivalent between IVO and IVM + groups.

\section{Variation in spindle dimensions between treatments and strains}

M-II oocytes labeled for chromatin, pericentrin and $\alpha / \beta$ tubulin were analyzed for spindle organization (Fig. 2) and from these images spindle size measurements which included spindle length, width, total area and mean spindle pole width were conducted, as summarized in Table 1.

In the IVO group, Black Swiss oocytes exhibited a significantly larger spindle area consistent with greater spindle width (Table 1 and Fig. 2C). This feature held true for IVM oocytes, since Black Swiss samples showed maximal spindle width and length in addition to broad spindle poles, and larger spindle total area as would be expected (Table 1 and Fig. 2c). At the other extreme, C57BL/6 and CF-1 oocytes exhibited smaller overall spindle areas after IVM due to their more compact spindle poles and slightly decreased overall length in comparison with the other strains (Table 1 and Fig. $2 b$ and d). In the the IVM + group, CF-1 oocytes showed wider spindles but with narrower poles and NU/NU oocytes displayed significantly narrower spindles (Table 1 and Fig. $2 d^{\prime}$ and $e^{\prime}$ ). In spite of these differences, total spindle area in IVM + oocytes was equivalent across strains. Overall, oocytes matured under basal IVM conditions exhibited more heterogeneity in spindle dimensions between strains when compared with IVO. Additionally, IVM + maturation conditions resulted in a decrease in the overall spindle size differences between strains when compared with IVM.

With reference to variations between maturation conditions within strains, a significant increase in spindle length, spindle pole width and total spindle area was evident for all strains studied as a result of IVM, when compared with IVO. However, spindle width was significantly increased only in B6D2F1 and Black Swiss oocytes. Overall, the ratio of spindle width at the equator to width at the poles decreased consistent with the transformation of spindles from a pointed/tapered shape in IVO oocytes to the more barrel-shaped IVM phenotype (Fig. 2). In contrast to the uniform increase in spindle dimensions observed for all strains in IVM with regard to IVO oocytes, oocyte maturation in IVM + conditions resulted in strainspecific variations in spindle size, except for the width of the spindle poles which was consistently intermediate between IVO and IVM in all strains. Spindle length in IVM + oocytes was similar to that in IVM oocytes, with the exception of the NU/NU strain, in which a significant decrease in length was observed. NU/NU and Black Swiss IVM + oocytes exhibited significantly narrower spindles than their IVO and IVM counterparts, while the opposite was observed in the CF-1 strain. In contrast, spindle width in B6D2F1 and C57BL/6 oocytes matured in IVM + was equivalent to both IVO and IVM oocytes. Finally, with regard to spindle total area, the effect of IVM + maturation conditions was again dependent on the strain. Specifically, spindle area was intermediate between IVM and IVO groups in B6D2F1 oocytes, similar to IVM in CF1 oocytes, but similar to IVO in the other three strains (C57BL/6, Black Swiss and NU/NU). Thus, although some strain-specific variations in spindle size were apparent, 


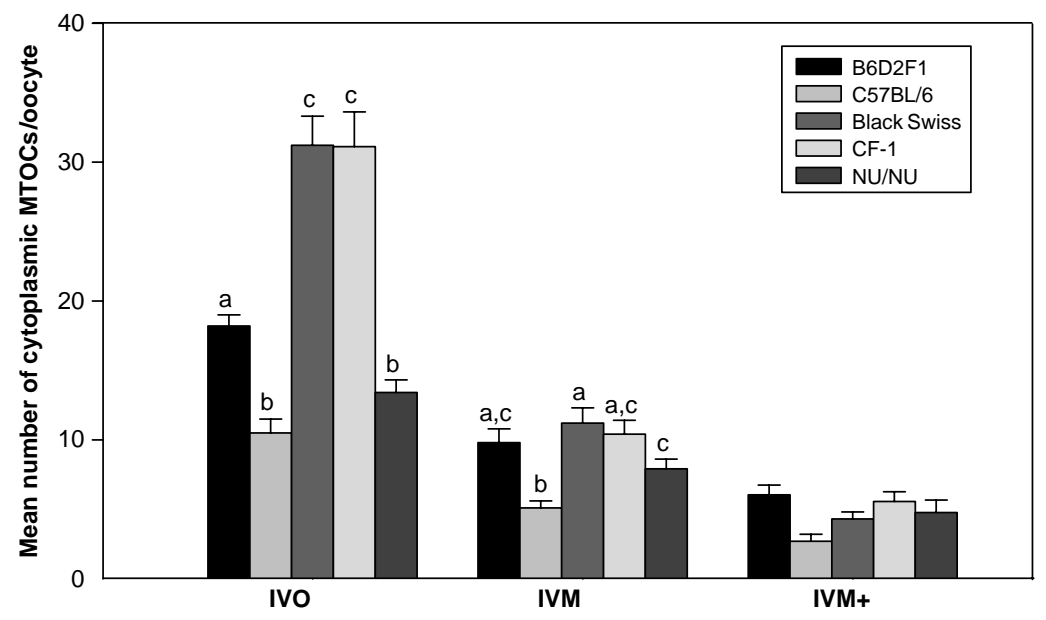

Figure 3 Mean number of cytoplasmic MTOCs in oocytes from five strains of mice matured under in vivo (IVO) or in vitro (IVM and IVM + ) conditions. Data represent the combined results of two replicate experiments for each maturation treatment, and the total number of oocytes analyzed for each strain were 10, 30 and 20 for IVO, IVM and IVM + respectively. Different letters at the top of the columns represent significant differences $(P<0.05)$ across strains within each maturation treatment (ANOVA). No differences were detected across strains in the mean number of cytoplasmic MTOCs in IVM + oocytes. the most consistent finding obtained from this analysis was that the $M$-II spindle area was increased for all strains as a result of IVM, when compared with IVO, and that maturation in a supplemented medium (IVM + ) tended to recapitulate the IVO spindle dimensions in a strain-dependent manner.

\section{Pericentrin organization and distribution at the spindle poles}

For each strain, the organization of pericentrin at the spindle poles highlighted variations in spindle size and shape evidenced above for IVO, IVM and IVM + matured oocytes (Table 1). Representative images from each group of oocytes are shown in Fig. 2 to illustrate the relationship between polar constriction and overall spindle size and shape. IVO oocytes typically exhibited spindles with focused poles, solitary pericentrin foci and symmetric halves as emphasized by equatorial chromatin disposition (Fig. 2A-E). An exception to this was the Black Swiss oocytes which usually displayed asymmetric half spindles in which the shorter half was associated with one pointed pole with a pericentrin focus and the longer half being less tapered with multiple foci at the pole (Fig. 2C). In fact, C-shaped polar aggregates of pericentrin were common in Black Swiss oocyte spindles and may explain their typically larger size and more asymmetric spindles (data not shown).

In general, pericentrin organization at the spindle poles varied in all strains as a function of IVM. IVM oocytes consistently displayed flat-poled and barrel-shaped spindles regardless of the strain, re-emphasizing the effect that culture can have on spindle phenotype. It appears further that spindle phenotype was related to the organization of pericentrin at the spindle poles as, overall, IVM oocytes tended to exhibit multiple pericentrin foci at the spindle poles (Fig. 2a-e), as described originally by Doxsey et al. (1994). Thus, the striking effect of culture on spindle properties was apparent at both the levels of spindle size and shape as well as the organization of pericentrin at the poles. In the case of the IVM + oocytes, an intermediate phenotype was evident with respect to spindle pole width and the presence of more compacted pericentrin at the poles relative to IVM oocytes but clearly less than that observed in IVO oocytes (Fig. $2 \mathrm{a}^{\prime}-\mathrm{e}^{\prime}$ ). Some strain variation in IVM + oocytes was evident, with B6D2F1 oocytes exhibiting the broadest spindle poles and CF-1 the most focused (Table 1). Again, the range of patterns showed that pericentrin in B6D2F1, C57BL/6 and Black Swiss oocytes organized as multiple foci $(\sim 50 \%)$ or single C-shaped aggregates $(\sim 50 \%)$, whereas in NU/NU and CF-1 oocytes solitary foci or C-shaped aggregates were most frequently observed at the spindle poles. Thus, variations in spindle size and shape were consistently associated with specific alterations in the organization of pericentrin at the spindle poles between strains and between different maturation groups.

\section{Cytoplasmic MTOC quantification between treatments and strains}

Having shown above that variations in spindle pole pericentrin distribution were associated with variable spindle pole widths and overall spindle shape, we next evaluated the distribution and number of cytoplasmic MTOCs across strains and maturation conditions. As shown in Fig. 3, IVO oocytes consistently exhibited increased numbers of cytoplasmic MTOCs across strains when compared with their IVM counterparts. Moreover, IVM + oocytes exhibited on average fewer cytoplasmic MTOCs relative to either IVM or IVO groups. Specifically, under IVO conditions, Black Swiss and CF-1 oocytes showed the greatest number of cytoplasmic MTOCs ( 30/oocyte), while B6D2F1 oocytes displayed an intermediate number $(\sim 20)$ and $\mathrm{C} 57 \mathrm{BL} / 6$ and NU/NU oocytes exhibited the lowest mean number $(\sim 10)$ of cytoplasmic MTOCs/oocyte. In the case of IVM, C57BL/6 oocytes showed the fewest cytoplasmic MTOCs ( $\sim 5 /$ oocyte) which may, in part, be related to delayed maturation in vivo. For IVM + oocytes, no differences were observed between strains but the overall number of 

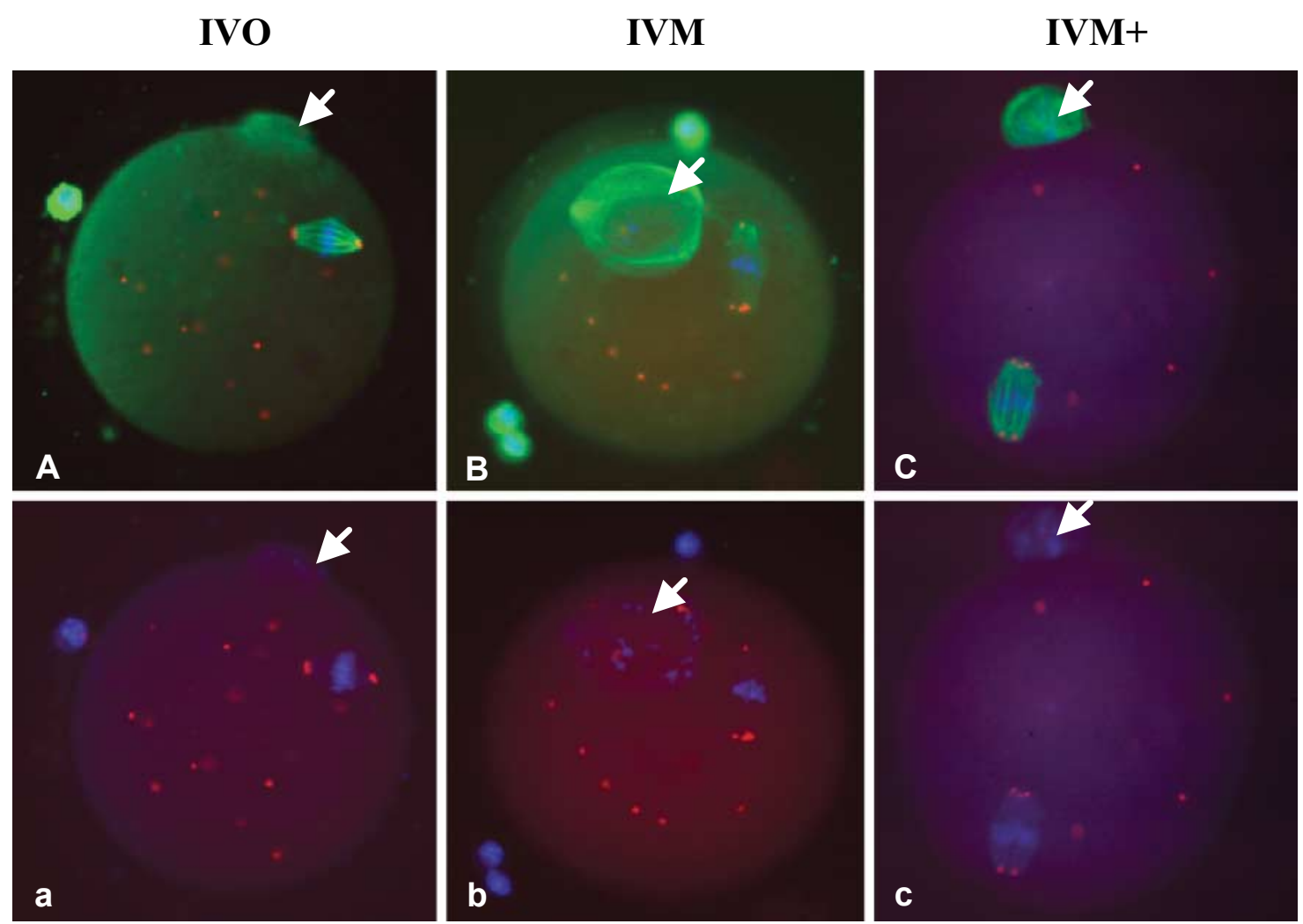

Figure 4 Cytoplasmic MTOCs and first polar body (PB) size in IVO, IVM and IVM + of NU/NU M-II oocytes. Tubulin (green), pericentrin (red) and chromosomes (blue) are depicted. Arrows indicate the position of the PB. (A-C) PB size and spindle shape and position in (A) IVO, (B) IVM and (C) IVM + oocytes. Correlative pericentrin and chromosome staining depicting a high number of cytoplasmic MTOCs in IVO oocytes, with small-sized PB, are shown in (a), IVM oocytes exhibit lower number of cytoplasmic MTOCs and bigger PBs (b) and oocytes cultured under IVM + conditions display the lowest number of cytoplasmic MTOCs and PBs of intermediate size (c). Note the presence of cytoplasmic MTOCs in the PB in IVM oocytes (b).

cytoplasmic MTOCs was significantly decreased when compared with IVM and IVO oocytes within the same strain. Interestingly, as shown above for the spindle size and shape, it appears that maturation under IVM + conditions diminished strain variance in MTOC number, with all samples exhibiting approximately five MTOCs/oocyte after IVM + . While variations in MTOC number between IVO, IVM and IVM + groups may be related to the amount of pericentrin associated with the spindle, other factors may affect the distribution of this material. For example, pericentrin-positive foci were found in the first polar body (Fig. 4). The presence of pericentrin material in the polar body was typically low in IVO oocytes (0-20\%; Fig. 4A and a), intermediate in IVM + oocytes (4-45\%; Fig. 4C and c) and high in IVM oocytes (40-79\%; Fig. 4B and b). It is also noteworthy that, regardless of the strain, IVM oocytes consistently exhibited larger polar bodies than their IVO counterparts. In fact, IVO oocytes typically exhibited polar bodies that were either small or absent altogether. Thus, the distribution and number of pericentrin-containing MTOCs was highly variable according to genetic background and a generalized decrease in MTOC number appeared to be associated with in vitro maturation. Whether increase in spindle size and appearance of MTOCs within the first polar body seen upon IVM and IVM + are interrelated consequences of culture remains to be established.

\section{Discussion}

While genetic background is well known to influence oocyte developmental competence in the mouse (Silver 1995), few studies have identified phenotypic traits in this species that might account for variations in fecundity. The present study analyzed microtubule patterning in oocytes from five strains of mice known to exhibit a wide range of fecundity. Oocytes collected following induced ovulation were remarkably similar across strains in terms of the centrosome-microtubule organization, with the exception of larger spindles in Black Swiss oocytes and significant variations in the number of cytoplasmic MTOCs, which were consistently higher in outbred mice. Given the known influence of the oocyte maturation milieu on the 
developmental potential of resulting embryos (Eppig \& O'Brien 1998, Moor et al. 1998, Trounson et al. 2001) we also compared IVO oocytes from each strain to oocytes matured in vitro under two conditions: using a basal (IVM) or a supplemented (IVM +) media. Our analysis has demonstrated a striking effect of in vitro maturation on the phenotype of the oocytes in terms of spindle, polar body and cytoplasmic MTOCs. While this effect has previously been reported in outbred CF-1 oocytes (Sanfins et al. 2003), the results of the present work in five different strains of mice and two different maturation media reinforced the idea that the environment in which the oocyte undergoes maturation is a critical factor in establishing and maintaining microtubule patterning in mouse oocytes, independent of the genetic background.

Our studies first showed that exogenous gonadotropins used to induce ovulation yielded fully mature oocytes that exhibited consistently tapered and small meiotic spindles, as previously observed by Sanfins et al. (2003) in CF-1 oocytes. With regard to nuclear maturation, IVM and IVM + oocytes reached $M$-II at slightly varying rates across strains. The only significant strain-specific difference resided in the low numbers of $\mathrm{M}$-II eggs for the inbred C57BL/6 strain after IVO in contrast to after IVM and IVM +. It is likely that variation in maturation kinetics reflects the low incidence of $\mathrm{M}$-II observed in vivo for C57BL/6 oocytes. Furthermore, the fact that C57BL/6 oocytes reach M-II at an elevated frequency after IVM or IVM + support the idea that the meiotic delay observed in vivo does not represent an intrinsic property of the oocyte, but possibly an effect of the follicular environment.

A notable feature of all in vitro matured oocytes was the transition in spindle phenotype from ones with tapered anastral poles (IVO) to larger barrel-shaped spindles (IVM) and an intermediate phenotype in the IVM + groups. Thus, some aspects of the processes underlying spindle morphogenesis must be susceptible to culture conditions and some degree of caution is warranted then in attributing significance to spindle shape variations when comparing in vivo with in vitro maturation conditions. Although every effort was made to normalize experimental conditions for sample preparation and analysis, and spindle properties are known to be affected by this (see Sanfins et al. 2003 for a discussion), the follicular milieu during ovulation was not being recapitulated under our culture conditions and must be a primary cause for the IVO versus IVM/IVM + differences detected. Similar to spindle shape, spindle dimensions were also modified by maturation conditions but, in this case, an effect of the oocyte genetic background was also evident. Thus, even though maturation under IVM conditions resulted in an increase in spindle dimensions in all strains when compared with IVO oocytes, it also resulted in a higher variability between strains than that observed in IVO oocytes for all the spindle parameters analyzed (length, width, pole width and total spindle area). For instance, while the only significant difference in spindle total area in IVO oocytes was the larger spindles in Black Swiss oocytes, IVM oocytes exhibited a wider range of sizes: larger spindles in Black Swiss oocytes, smaller spindles in C57BL/6 and CF-1 oocytes and spindles of intermediate size in B6D2F1 and NU/NU oocytes. When compared with IVM, variability in spindle dimensions between strains was reduced after maturation in IVM + conditions, as significant differences among strains were only evident in spindle width and pole width. However, strain-specific differences in all spindle parameters analyzed were evident in this case when comparing IVM + with IVO and IVM oocytes, except for the width of the spindle poles.

Genetic variations in mouse oocytes have been described by others. Strain-specific rates of maturation were reported previously for both in vivo and in vitro matured oocytes (Polanski 1986, 1997), and whether differences in the synthesis of key cell cycle factors such as cyclins (Polanski et al. 1998), c-mos (Choi et al. 1996, Verlhac et al. 2000) or cdc25b (Lincoln et al. 2002) explain the incidence of $\mathrm{M}$-II oocytes in the inbred C57BL/6 or other variations remains an interesting possibility. The fact that the overall incidence of maturation to M-II was lower with IVM oocytes when compared with IVO oocytes most likely reflects the greater developmental heterogeneity of oocytes retrieved after eCG. While this heterogeneity is in accordance with previous reports (Combelles \& Albertini 2003), our results begin to uncover phenotypic variations intrinsic to the genotype that are manifest as alterations in microtubule patterning during oocyte maturation.

Our analysis of spindle dimensions revealed significant differences, with small pointed spindles in IVO oocytes when compared with larger less-tapered spindles in IVM and IVM + oocytes. Furthermore, IVM + spindles exhibited characteristics intermediate between IVM and IVO spindles in being both smaller and neither flat nor pointed at the poles. This finding suggested that mechanisms of spindle assembly may differ under in vitro and in in vivo conditions and further that forces shaping the spindle pole are being regulated differently in vivo.

Another key phenotypic variant identified in this work pertains to the disposition of the centrosomal protein pericentrin at both the spindle poles and in the oocyte cortex. Pericentrin organization has been described during in vitro maturation in oocytes originating from outbred mice (Doxsey et al. 1994, Carabatsos et al. 2000, Combelles \& Albertini 2001). While confirming the arrangement of pericentrin at the poles of IVM spindles across five strains of mice, our work further describes the differential distribution of pericentrin at the spindle poles of IVO and IVM + oocytes. The pointed spindles of IVO oocytes usually displayed a single focus of pericentrin while the broad, barrel-shaped spindles of IVM oocytes exhibited multiple and distinct foci at the 
poles. Here again, pericentrin organization at the poles of IVM + spindles resembled more closely patterns observed after IVO than after IVM. Furthermore, in the case of the outbred Black Swiss strain, alterations in spindle shape were observed concomitantly with changes in pericentrin organization at the spindle poles. While the mechanisms underlying spindle assembly, maintenance and function remain unknown in mammalian oocytes, the present study points towards important microtubule and pericentrin differences under varying maturation conditions and genetic backgrounds. It may be speculated here that spindles in in vivo ovulated oocytes are under more tension when compared with spindles in in vitro matured ones, which display relaxed organization with respect to microtubules and pericentrin at the poles.

The distribution of centrosomal proteins involved in microtubule microtubule patterning, including pericentrin and $\alpha$-tubulin, which organize meiotic spindle microtubules and microtubules associated with the oocyte cortex, is known to change according to the stage of meiotic cell cycle progression in mice (Messinger \& Albertini 1991, Albertini 1992, Combelles \& Albertini 2001, Sanfins et al. 2003). Mouse oocytes are characterized by the unique presence of cytoplasmic centrosome aggregates (Maro et al. 1985) that nucleate microtubules upon anaphase of both meiosis-I or meiosis-II (Combelles \& Albertini 2001). It has been proposed to be a maternal store of MTOCs for later embryonic mitoses (Maro et al. 1985, Houliston et al. 1987, Schatten 1994). Although speculative, it is conceivable that differences in the number of cytoplasmic MTOCs between IVO, IVM, and IVM + oocytes and across strains illustrate the pool of centrosomal material available in each oocyte type. Our work suggests that loss to the polar body or excessive spindle integration could be related to differences in MTOC number seen in IVM and IVM + oocytes when compared with IVO. If this were the case, then the limited development of embryos seen after in vitro maturation, coupled with our findings of a decrease in MTOC number, might explain deficiencies in cell cycle timing (Sanfins et al. 2003) or cell polarity determination (Liu et al. 2003, Albertini \& Barrett 2004) seen after in vitro maturation.

Numerous mouse strains are routinely being used to generate gene knockout, transgenic, nuclear transfercloned and new mutagenesis-induced lines. Despite the expanding utility of genetically modified mouse models, relatively little effort has been expended in defining the phenotypic properties of murine oocytes that would be of predictive value in defining the maternal origins of oocyte developmental competence. Additionally, the notion that cytoplasmic properties of the M-II oocyte influence reprogramming events after nuclear transfer (Fulka et al. 2001, Liu et al. 2001) emphasizes the need to establish markers of oocyte cytoplasmic quality. With regard to microtubule patterning as a phenotypic marker, previous results in CF- 1 oocytes (Combelles \& Albertini 2003) and the present analysis on a limited number of strains establish feasibility and utility for this purpose. Moreover, most laboratories employ a single strain in their studies, thereby possibly restricting the value of their findings to a solitary genetic background. In conclusion, our studies identifying microtubule patterning variations in five strains of mice together with wellrecognized epigenetic factors like culture, suggest that both genetic attributes and environmental modulators must be taken into account in studies aimed at understanding the mechanisms of meiotic spindle assembly, control of the meiotic cell cycle and determinants of oocyte quality in general.

\section{Acknowledgements}

The authors thank Dr Phyllis Mann for advice on statistical analysis, and Lynda McGinnis and Susan Barrett for reading the manuscript and making helpful suggestions. We acknowledge Charles River Laboratories for their generous provision of animals used in this work. This work was supported by a Fulbright grant sponsored by the Spanish Ministry of Education, Culture and Sports (to E I) and grants from Fundaçao para a Ciencia e Tecnologia (FCT) SFRH/BD/2757/2000 (to A S), USDA-NRI 2001-35203-09966 (to E W O and D F A) and the ESHE Fund and March of Dimes Birth Defects Foundation 01-248 (to D F A).

\section{References}

Albertini DF 1992 Cytoplasmic microtubular dynamics and chromatin organization during mammalian oogenesis and oocyte maturation. Mutation Research 296 57-68.

Albertini DF \& Barrett SL 2004 The developmental origins of mammalian oocyte polarity. Seminars in Cell and Developmental Biology 15 599-606.

Carabatsos MJ, Combelles CM, Messinger SM \& Albertini DF 2000 Sorting and reorganization of centrosomes during oocyte maturation in the mouse. Microscopy Research and Technique 49 435-444.

Chen L, Wert S, Hendrix E, Russell P, Cannon M \& Larsen W 1990 Hyaluronic acid synthesis and gap junction endocytosis are necessary for normal expression of the cumulus mass. Molecular Reproduction and Development 26 236-247.

Choi T, Fukasawa K, Zhou R, Tessarollo L, Borror K, Resau J \& Vande Woude GF 1996 The Mos/mitogen-activated protein kinase (MAPK) pathway regulates the size and degradation of the first polar body in maturing mouse oocytes. PNAS 93 7032-7035.

Combelles CM \& Albertini DF 2001 Microtubule patterning during meiotic maturation in mouse oocytes is determined by cell cyclespecific sorting and redistribution of gamma-tubulin. Developmental Biology 239 281-294.

Combelles CM \& Albertini DF 2003 Assessment of oocyte quality following repeated gonadotropin stimulation in the mouse. Biology of Reproduction $\mathbf{6 8} 812-821$.

Dell'Aquila M, Gaillaud M, Maritato F, Martoriati A, Gerard N, Aiudi G, Minoia P \& Goudet G 2004 Cumulus expansion, nuclear maturation and connexin 43, cyclooxygenase-2 and FSH receptor mRNA expression in equine cumulus-oocyte complexes cultured in vitro in the presence of FSH and precursors for hyaluronic acid synthesis. Reproductive Biology and Endocrinology 2 44-57. 
Doxsey SJ, Stein P, Evans L, Calarco PD \& Krischner M 1994 Pericentrin, a highly conserved centrosome protein involved in microtubule organization. Cell 76 639-650.

Eichenlaub-Ritter U \& Peschke M 2002 Expression in in vivo and in vitro growing and maturing oocytes: focus on regulation of expression at the translational level. Human Reproduction Update 8 $21-41$.

Eppig JJ 1979 FSH stimulates hyaluronic acid synthesis by oocytecumulus cell complexes from mouse preovulatory follicles. Nature $281483-484$.

Eppig JJ 1980 Role of serum in FSH stimulated cumulus expansion by mouse oocyte-cumulus cells complexes in vitro. Biology of Reproduction 22 629-633.

Eppig JJ \& O'Brien MJ 1998 Comparison of preimplantation developmental competence after mouse oocyte growth and development in vitro and in vivo. Theriogenology 49 415-422.

Eppig JJ, O'Brien M \& Wigglesworth K 1996 Mammalian oocyte growth and development in vitro. Molecular Reproduction and Development 44 260-273.

Eppig J, Hosoe M, O'Brien M, Pendola F, Requena A \& Watanabe S 2000 Conditions that affect acquisition of developmental competence by mouse oocytes in vitro: $\mathrm{FSH}$, insulin, glucose and ascorbic acid. Molecular and Cellular Endocrinology 163 109-116.

Fulka J Jr, Loi P, Ledda S, Moor RM \& Fulka J 2001 Nucleus transfer in mammals: how the oocyte cytoplasm modifies the transferred nucleus. Theriogenology 55 1373-1380.

Gao S, Czirr E, Chung YG, Han Z \& Latham KE 2004 Genetic variation in oocyte phenotype revealed through parthenogenesis and cloning: correlation with differences in pronuclear epigenetic modification. Biology of Reproduction 70 1162-1170.

Houliston E, Pickering SJ \& Maro B 1987 Redistribution of microtubules and pericentriolar material during the development of polarity in mouse blastomeres. Journal of Cell Biology 104 1299-1308.

Ibáñez E, Albertini DF \& Overström EW 2005 Effect of genetic background and activating stimulus on the timing of meiotic cell cycle progression in parthenogenetically activated mouse oocytes. Reproduction 129 27-38.

Kaleta E 1977 Influence of genetic factors on the fertilization of mouse ova in vitro. Journal of Reproduction and Fertility $\mathbf{5 1}$ $375-381$.

Lincoln AJ, Wickramasinghe D, Stein P, Schultz RM, Palko ME, De Miguel MP, Tessarollo L \& Donovan PJ 2002 Cdc25b phosphatase is required for resumption of meiosis during oocyte maturation. Nature Genetics 30 446-449.

Liu H, Krey LC, Zhang J \& Grifo JA 2001 Ooplasmic influence on nuclear function during the metaphase II-interphase transition in mouse oocytes. Biology of Reproduction 65 1794-1799.

Liu M, Sims D, Calarco P \& Talbot P 2003 Biochemical heterogeneity, migration, and pre-fertilization release of mouse oocyte cortical granules. Reproductive Biology and Endocrinology 177.

Lucifero D, Mertineit C, Clarke HJ, Bestor TH \& Trasler JM 2002 Methylation dynamics of imprinted genes in mouse germ cells. Genomics 79 530-538.

Luckett DC \& Mukherkee AB 1986 Embryonic characteristics in superovulated mouse strains. Journal of Heredity 77 39-42.

McLaren A \& Bowman P 1973 Genetic effects on the timing of early development in the mouse. Journal of Embryology and Experimental Morphology $30491-498$.

Maro B, Howlett SK \& Webb M 1985 Non-spindle microtubule organizing centers in metaphase II-arrested mouse oocytes. Journal of Cell Biology 101 1665-1672.

de Matos D, Furnus C, Moses D \& Balsassarre H 1995 Effect of cysteamine on glutathione level and developmental capacity of bovine oocyte matured in vitro. Molecular Reproduction and Development 42 432-436.

de Matos D, Furnus C, Moses D, Martinez A \& Matkovic M 1996 Stimulation of glutathione synthesis of in vitro matured bovine oocytes and its effect on embryo development and freezability. Molecular Reproduction and Development 45 451-457. de Matos D, Nogueira D, Cortvrindt R, Herrera C, Adriaenssens T, Pasqualini RS \& Smitz J 2003 Capacity of adult and prepubertal mouse oocytes to undergo embryo development in the presence of cysteamine. Molecular Reproduction and Development 64 214-218.

Merriman JA, Whittingham DG \& Carroll J 1998 The effect of follicle stimulating hormone and epidermal growth factor on the developmental capacity of in vitro matured mouse oocytes. Human Reproduction 13 690-695.

Messinger SM \& Albertini DF 1991 Centrosome and microtubule dynamics during meiotic progression in the mouse oocyte. Journal of Cell Science 100 289-298.

Moor RM \& Dai Y 2001 Maturation of pig oocytes in vivo and in vitro. Reproduction 58 (Suppl) 91-104.

Moor RM, Dai Y, Lee C \& Fulka J Jr 1998 Oocyte maturation and embryonic failure. Human Reproduction Update 4 223-236.

Niemann H \& Wrenzycki C 2000 Alterations of expression of developmentally important genes in preimplantation bovine embryos by in vitro culture conditions: implications for subsequent development. Theriogenology 53 21-34.

Nishi Y, Takeshita T, Sato K \& Araki T 2003 Change of the mitochondrial distribution in mouse ooplasm during in vitro maturation. Journal of Nippon Medical School 70 408-415.

Perreault S, Barbee R \& Slott V 1988 Importance of glutathione in the acquisition and maintenance of sperm nuclear decondensing activity in maturing hamster oocytes. Developmental Biology 125 $181-186$.

Polanski Z 1986 In vivo and in vitro maturation rate of oocytes from two strains of mice. Journal of Reproduction and Fertility $\mathbf{7 8}$ $103-109$.

Polanski Z 1997 Genetic background of the differences in timing of meiotic maturation in mouse oocytes: a study using recombinant inbred strains. Journal of Reproduction and Fertility 109 109-114.

Polanski Z, Ledan E, Brunet S, Louvet S, Verlhac MH, Kubiak JZ \& Maro B 1998 Cyclin synthesis controls the progression of meiotic maturation in mouse oocytes. Development 125 4989-4997.

Rodriguez KF \& Farin CE 2004 Gene transcription and regulation of oocyte maturation. Reproduction, Fertility and Development $\mathbf{1 6}$ $55-67$.

Sanfins A, Lee GY, Plancha CE, Overstrom EW \& Albertini DF 2003 Distinctions in meiotic spindle structure and assembly during in vitro and in vivo maturation of mouse oocytes. Biology of Reproduction 69 2059-2067.

Sanfins A, Plancha CE, Overstrom EW \& Albertini DF 2004 Meiotic spindle morphogenesis in in vivo and in vitro matured mouse oocytes: insights into the relationship between nuclear and cytoplasmic quality. Human Reproduction 19 2889-2899.

Schatten G 1994 The centrosome and its mode of inheritance: the reduction of the centrosome during gametogenesis and its restoration during fertilization. Developmental Biology 165 299-335.

Schultz RM, LaMarca MJ \& Wassarman PM 1978 Absolute rates of protein synthesis during meiotic maturation of mammalian oocytes in vitro. PNAS 75 4160-4164.

Silver LM 1995 Reproduction and Breeding. In Mouse Genetics. Concepts and Applications, pp 62-63. New York: Oxford University Press.

Spearow JL 1988 Characterization of genetic differences in hormoneinduced ovulation rate in mice. Journal of Reproduction and Fertility 82 799-806.

Spearow JL, Doemeny P, Sera R, Leffler R \& Barkley M 1999 Genetic variation in susceptibility to endocrine disruption by estrogen in mice. Science 285 1259-1261.

Steeves CL, Lane M, Bavister BD, Phillips KP \& Baltz JM 2001 Differences in intracellular $\mathrm{pH}$ regulation by $\mathrm{Na}(+) / \mathrm{H}(+)$ antiporter among two-cell mouse embryos derived from females of different strains. Biology of Reproduction 65 14-22.

Suzuki O, Asano T, Yamamoto Y, Takano K \& Koura M 1996 Development in vitro of preimplantation embryos from 55 
mouse strains. Reproduction, Fertility and Development 8 975-980.

Tatemoto H, Ootaki K, Shigeta K \& Muto N 2001 Enhancement of developmental competence after in vitro fertilization of porcine oocytes by treatment with ascorbic acid 2-O-alpha-glucoside during in vitro maturation. Biology of Reproduction 65 1800-1806.

Trounson A, Anderiesz C \& Jones G 2001 Maturation of human oocytes in vitro and their developmental competence. Reproduction $12151-75$
Verlhac MH, Lefebvre C, Guillaud P, Rassinier P \& Maro B 2000 Asymmetric division in mouse oocytes: with or without Mos. Current Biology $101303-1306$.

Received 5 November 2004

First decision 12 January 2005

Revised manuscript received 4 August 2005

Accepted 19 August 2005 